IAU Colloquium 164: Radio Emission from Galactic and Extragalactic Compact Sources

ASP Conference Series, Vol. 144, 1998

J. A. Zensus, G. B. Taylor, \& J. M. Wrobel (eds.)

\title{
The Identification of EGRET Sources with Flat-Spectrum Radio Sources
}

J. R. Mattox

Astronomy Department, Boston University, Boston, MA 02215, U.S.A.

Abstract. We provide a table of 42 blazars which we expect to be robust identifications of EGRET sources. The table includes the lensed source, PKS 1830-211 which we identify with a previously unidentified EGRET source.

\section{Introduction}

A careful analysis of the identifications of EGRET sources with radio sources is in order because the source of the $\gamma$-ray emission is not well located. An analysis of all EGRET sources off the Galactic plane in the 2nd EGRET catalog and its extension (Thompson et al. 1995, 1996) has been published by Mattox et al. (1997).

\section{The Catalog}

The catalog of 42 sources for which the confidence of the identification exceeds $\sim 90 \%$ is given in the table. Column 1 is the epoch 1950 position name of the radio source. Column 2 gives the $5 \mathrm{GHz}$ flux density and column 3 the $\mathrm{cm}$ radio spectral index, $S(\nu) \propto \nu^{\alpha}$. Column 4 gives the correlated VLBI flux density at $2.29 \mathrm{GHz}$ from Preston et al. (1985). Column 5 gives the probability that the identification is corrected based on EGRET data and radio data. In some cases increased confidence results from variability of the $\gamma$-ray flux or a high VLBI flux density. See Mattox et al. (1997) for details. Column 6 gives the peak flux detected by EGRET, and column 7 gives the EGRET photon spectral index, $d F(E) \propto E^{-\gamma} d E$. During the analysis done to produce this catalog, Mattox et al. (1997) found that:

1) It appears that only the blazar class of AGN is being detected.

2) The EGRET sources have higher VLBI flux densities than the parent population of flat radio spectrum sources.

3) The peak $\gamma$-ray flux of a blazar is correlated with its average $5 \mathrm{GHz}$ radio flux. An even better correlation is seen between $\gamma$-ray flux and VLBI flux.

\section{References}

Mattox, J. R., et al. 1997. $A p J, 481,95-115$.

Preston, R. A., et al. 1985. $A J, 90,1599-1641$.

Thompson, D. J., et al. 1995. ApJS, 101, 259-286.

Thompson, D. J., et al. 1996. ApJS, 107, 227-237. 


\begin{tabular}{|c|c|c|c|c|c|c|}
\hline $\begin{array}{l}\text { Name } \\
\text { B1950 }\end{array}$ & $S_{5}$ & $\alpha$ & $S_{V L B I}$ & $p(i d \mid r)$ & $\begin{array}{c}\text { Peak Flux } \\
10^{-6} \mathrm{~cm}^{-2} \mathrm{~s}^{-1} \\
\text { E }>100 \mathrm{MeV}\end{array}$ & $\begin{array}{c}\gamma \text {-Ray Spectral } \\
\text { Index }\end{array}$ \\
\hline $0202+149$ & 2714 & -0.4 & $600 \pm 100$ & 0.994 & $0.26 \pm 0.06$ & $2.5 \pm 0.1$ \\
\hline $0208-512$ & 3198 & -0.2 & $2230 \pm 110$ & 0.996 & $1.10 \pm 0.07$ & $1.7 \pm 0.1$ \\
\hline $0234+285$ & 3356 & 0.3 & $1600 \pm 100$ & 0.95 & $0.16 \pm 0.04$ & $2.7 \pm 0.3$ \\
\hline $0235+164$ & 1955 & -0.1 & $1800 \pm 200$ & 0.95 & $0.82 \pm 0.09$ & $2.0 \pm 0.2$ \\
\hline $0336-019$ & 3014 & 0.3 & $1400 \pm 100$ & 0.997 & $4 \pm 1$ & $2.4 \pm 0.3$ \\
\hline $0420-014$ & 6992 & 1.1 & $610 \pm 50$ & 0.9995 & $0.50 \pm 0.10$ & $1.9 \pm 0.3$ \\
\hline $0440-003$ & 1084 & 0.6 & $820 \pm 40$ & 0.91 & $0.84 \pm 0.12$ & $1.8 \pm 0.2$ \\
\hline $0458-020$ & 3317 & 1.0 & $920 \pm 80$ & 0.96 & $0.31 \pm 0.09$ & $2.5 \pm 0.4$ \\
\hline $0521-365$ & 8180 & -0.7 & $990 \pm 90$ & 0.9989 & $0.21 \pm 0.06$ & $2.2 \pm 0.4$ \\
\hline $0528+134$ & 2978 & 0.3 & $500 \pm 80$ & 0.999 & $2.95 \pm 0.33$ & $2.6 \pm 0.1$ \\
\hline $0537-441$ & 4805 & 0.4 & $2030 \pm 90$ & 0.998 & $0.36 \pm 0.09$ & $2.0 \pm 0.2$ \\
\hline $0716+714$ & 810 & 0.0 & $600 \pm 50$ & 0.78 & $0.53 \pm 0.13$ & $1.9 \pm 0.2$ \\
\hline $0735+178$ & 2146 & 0.1 & $600 \pm 100$ & 0.96 & $0.30 \pm 0.12$ & $2.5 \pm 0.5$ \\
\hline $0827+243$ & 670 & 0.0 & $800 \pm 100$ & 0.34 & $0.26 \pm 0.06$ & $2.2 \pm 0.4$ \\
\hline $0829+046$ & 2105 & 0.7 & $500 \pm 100$ & 0.86 & $0.20 \pm 0.06$ & $2.5 \pm 0.5$ \\
\hline $0836+710$ & 2436 & -0.4 & $370 \pm 30$ & 0.79 & $0.45 \pm 0.11$ & $2.4 \pm 0.2$ \\
\hline $0954+556$ & 2260 & -0.2 & $<100$ & 0.989 & $0.11 \pm 0.04$ & $1.8 \pm 0.3$ \\
\hline $0954+658$ & 1419 & 0.6 & $430 \pm 30$ & 0.992 & $0.14 \pm 0.04$ & $1.7 \pm 0.2$ \\
\hline $1101+384$ & 712 & -0.1 & $320 \pm 40$ & 0.70 & $0.23 \pm 0.08$ & $1.7 \pm 0.2$ \\
\hline $1127-145$ & 4209 & 0. & $1490 \pm 70$ & 0.90 & $0.93 \pm 0.23$ & $2.7 \pm 0.4$ \\
\hline $1156+295$ & 1542 & -0.1 & $430 \pm 50$ & 0.94 & $2.29 \pm 0.55$ & $2.0 \pm 0.5$ \\
\hline $1219+285$ & 968 & 0. & $400 \pm 40$ & 0.74 & $0.28 \pm 0.09$ & $1.3 \pm 0.4$ \\
\hline $1222+216$ & 1261 & -0.4 & $330 \pm 40$ & 0.40 & $0.83 \pm 0.20$ & $1.9 \pm 0.4$ \\
\hline $1226+023$ & 44940 & -0.1 & $1510 \pm 70$ & 0.9995 & $0.24 \pm 0.05$ & $2.4 \pm 0.1$ \\
\hline $1253-055$ & 11192 & -0.1 & $3700 \pm 300$ & 0.999991 & $4.5 \pm 0.6$ & $1.9 \pm 0.1$ \\
\hline $1406-076$ & 1080 & 0.2 & $640 \pm 50$ & 0.86 & $1.44 \pm 0.26$ & $2.0 \pm 0.1$ \\
\hline $1424-418$ & 2597 & 0.0 & $370 \pm 40$ & 0.98 & $0.55 \pm 0.17$ & $2.6 \pm 0.4$ \\
\hline $1510-089$ & 3000 & 0.0 & $2300 \pm 130$ & 0.98 & $0.48 \pm 0.18$ & $2.6 \pm 0.4$ \\
\hline $1606+106$ & 1688 & 0.0 & $490 \pm 50$ & 0.95 & $0.60 \pm 0.13$ & $2.2 \pm 0.3$ \\
\hline $1611+343$ & 2483 & -0.1 & $1200 \pm 100$ & 0.95 & $0.55 \pm 0.13$ & $2.0 \pm 0.2$ \\
\hline $1622-253$ & 3449 & 0.7 & $150 \pm 20$ & 0.998 & $0.43 \pm 0.07$ & $2.3 \pm 0.2$ \\
\hline $1622-297$ & 1920 & 0.1 & $2000 \pm 200$ & 0.996 & $17 \pm 3$ & $1.9 \pm 0.2$ \\
\hline $1633+382$ & 3198 & 0.4 & $1300 \pm 100$ & 0.9996 & $1.8 \pm 0.3$ & $1.9 \pm 0.1$ \\
\hline $1730-130$ & 6991 & 0.8 & $1420 \pm 60$ & 0.988 & $1.37 \pm 0.43$ & $2.4 \pm 0.3$ \\
\hline $1739+522$ & 1134 & -0.4 & $650 \pm 70$ & 0.61 & $0.54 \pm 0.11$ & $2.2 \pm 0.4$ \\
\hline $1830-211$ & 7920 & -0.3 & & 0.98 & $0.70 \pm 0.19$ & $2.7 \pm 0.3$ \\
\hline $1908-201$ & 2053 & 0 . & $460 \pm 50$ & 0.95 & $0.29 \pm 0.10$ & $2.5 \pm 0.2$ \\
\hline $2052-474$ & 2026 & 0. & $380 \pm 40$ & 0.90 & $0.25 \pm 0.08$ & $2.4 \pm 0.4$ \\
\hline $2200+420$ & 3571 & -0.2 & $720 \pm 60$ & 0.993 & $0.40 \pm 0.12$ & $2.2 \pm 0.4$ \\
\hline $2209+236$ & 1194 & 0.7 & $550 \pm 50$ & 0.81 & $0.13 \pm 0.04$ & $2.8 \pm 0.5$ \\
\hline $2230+114$ & 3765 & -0.5 & $600 \pm 100$ & 0.998 & $0.53 \pm 0.22$ & $2.6 \pm 0.2$ \\
\hline $2251+158$ & 15859 & 0.1 & $3400 \pm 300$ & 0.9998 & $1.32 \pm 0.21$ & $2.2 \pm 0.1$ \\
\hline
\end{tabular}

